\title{
Erratum to: HDAC6 activity is a non- oncogene addiction hub for inflammatory breast cancers
}

Preeti Putcha ${ }^{4 \dagger}$, Jiyang $\mathrm{Yu}^{2 \dagger}$, Ruth Rodriguez-Barrueco ${ }^{1 \dagger}$, Laura Saucedo-Cuevas ${ }^{1}$, Patricia Villagrasa ${ }^{5}$, Eva Murga-Penas ${ }^{5}$, Steven N. Quayle ${ }^{6}$, Min Yang ${ }^{6}$, Veronica Castro ${ }^{5}$, David Llobet-Navas ${ }^{1}$, Daniel Birnbaum ${ }^{9}$, Pascal Finetti ${ }^{9}$, Wendy A. Woodward ${ }^{8}$, François Bertucci ${ }^{9}$, Mary L. Alpaugh ${ }^{7 *}$, Andrea Califano ${ }^{2,3,4^{*}}$ and Jose Silva ${ }^{1 *}$

\section{Erratum}

After the publication of this article [1] the authors noticed two small mistakes in the affiliations listings

1) Dr. Ruth Rodriguez should be marked as an equal contributor alongside Dr. Putcha and Dr. Yu.

2) Dr. Ruth Rodriguez's correct affiliation is 'Department of Pathology, Icahn School of Medicine at Mount Sinai , New York, NY 10029-6574, USA' and not 'Department of Radiation Oncology, The University of Texas MD Anderson Cancer Center, Houston, TX, USA' as the original article states.

\begin{abstract}
Author details
'Department of Pathology, Icahn School of Medicine at Mount Sinai, New York, NY 10029-6574, USA. ${ }^{2}$ Department of Biomedical Informatics, Department of Systems Biology, Center for Computational Biology and Bioinformatics, Herbert Irving Comprehensive Cancer Center, Columbia University, New York, NY 10032, USA. ${ }^{3}$ Department of Biochemistry and Molecular Biophysics, Institute for Cancer Genetics, Columbia University, New York, NY 10032, USA. ${ }^{4}$ Herbert Irving Comprehensive Cancer Center, Columbia University, 1130 St. Nicholas Avenue, New York, NY 10032, USA. ${ }^{5}$ Department of Pathology, Columbia University Medical Center, 630 West168th Street, New York, NY 10032, USA. ${ }^{6}$ Acetylon Pharmaceuticals, Inc., 70 Fargo St, Suite 205, Boston, MA 02210, USA. 'Department of Surgery, Memorial Sloan Kettering Cancer Center, New York, NY 10065, USA. ${ }^{8}$ Department of Radiation Oncology, The University of Texas MD Anderson Cancer Center, Houston, TX, USA. ${ }^{9}$ Centre de Recherche en Cancérologie de Marseille, Institut Paoli-Calmettes, Aix-Marseille Université, Marseille, France.
\end{abstract}

Received: 5 April 2017 Accepted: 7 April 2017 Published online: 19 April 2017

\section{Reference}

1. Putcha P, Yu J, Rodriguez-Barrueco R, Saucedo-Cuevas L, Villagrasa P, Murga-Penas E, Quayle SN, Yang M, Castro V, Llobet-Navas D, Birnbaum D, Finetti P, Woodward WA, Bertucci F, Alpaugh ML, Califano A, Silva J. HDAC6 activity is a non-oncogene addiction hub for inflammatory breast cancers. Breast Cancer Res. 2015;17:149. doi:10.1186/s13058-015-0658-0.

\footnotetext{
*Correspondence: alpaughm@mskcc.org; califano@c2b2.columbia.edu; jose.silva@mssm.edu

${ }^{\dagger}$ Equal contributors

${ }^{7}$ Department of Surgery, Memorial Sloan Kettering Cancer Center, New York, NY 10065, USA

${ }^{2}$ Department of Biomedical Informatics, Department of Systems Biology, Center for Computational Biology and Bioinformatics, Herbert Irving Comprehensive Cancer Center, Columbia University, New York, NY 10032, USA

'Department of Pathology, Icahn School of Medicine at Mount Sinai, New York, NY 10029-6574, USA
} 